\title{
Crystal engineering with copper and melamine ${ }^{\dagger}$
}

\author{
Ignacio Bernabé Vírseda ${ }^{\ddagger}$ Shiraz Ahmed Siddiqui, " Alexander Prado-Roller ${ }^{\S}$ \\ Michael Eisterer, $\|$ and Hidetsugu Shiozawa*,, , \\ $\ddagger J$. Heyrovsky Institute of Physical Chemistry, Czech Academy of Sciences, Dolejskova 3, \\ 182 23 Prague 8, Czech Republic \\ IFaculty of Physics, University of Vienna, Boltzmanngasse 5, 1090 Vienna, Austria \\ §Institute of Inorganic Chemistry, Faculty of Chemistry, University of Vienna, Währinger \\ Straße 42, Austria \\ ||Atominstitut, TU Wien, Stadionallee 2, 1020 Vienna, Austria
}

E-mail: E-mail:hidetsugu.shiozawa@univie.ac.at,hide.shiozawa@jh-inst.cas.cz Phone: +43-1-4277-72601,+420-26605-3755. Fax: +43-1-4277-9726

\begin{abstract}
Coordination complex and polymer are central in inorganic and material chemistry as a variety of both metal centers and coordination geometries lead to a diverse range of interesting properties. Here, size and structure control of gems-like quality monocrystals are demonstrated at room temperature. From the same set of precursors, but the copper-to-melamine molar ratio is adjusted to synthesize either a novel coordination complex of dinuclear copper and melamine (Cu2M1) or a barely-studied coordination polymer of zigzag copper-chlorine chains (Cu4M1). Crystals of the former are dark green and squared with the size up to $350 \mu \mathrm{m}$ across. The latter is light green and octagonal as large as $5 \mathrm{~mm}$ across. The magnetic properties of both crystals reflect their
\end{abstract}

\footnotetext{
${ }^{\dagger}$ A footnote for the title
} 
low-dimensional arrangements of copper. The magnetic susceptibility of Cu2M1 is well modelled with a spin-1/2 dimer and that of Cu4M1 with a spin-1/2 one-dimensional Ising chain. Controlled synthesis of such quality magnetic crystals is a prerequisite for various magnetic and magneto-optical applications.

\section{Introduction}

Advanced crystal engineering continues to draw the attention of the scientific community. This discipline is leading to new structures of organometallic complexes by the design of the coordination between organic ligands and metal ions. Organometallic complexes are the molecules composed of a centrally located metal or a cluster of metals bonded covalently at least to one carbon atom of the peripheral organic compounds called ligands. If the ligand interacts with the metal through a heteroatom (an atom other than carbon or hydrogen), it is called "coordination complex". ${ }^{1}$ Furthermore, metal ions and ligands are able to form polymeric structures, such as coordination polymers and metal organic frameworks (MOFs). Coordination polymers are those organometallic polymeric structures composed of metal ions being linked by organic ligands to form one-, two-, or three-dimensional structures. And MOFs are mesoporous coordination polymers ${ }^{234} .{ }^{5}$ Organometallic chemistry deals with optimization of synthesis conditions to reach defined and stable organic building blocks with outstanding properties. ${ }^{6}$ All these organometallic materials are used in a great deal of applications such as energy transfer, gas storage and separation, heterogeneous catalysis, proton conduction, biomedical applications and chemical sensing ${ }^{2}{ }^{7}$ Molecular magnets based on organometallic compounds play an essential role in information storage in quantum computing. Organometallic single crystals with clustered Mn and Fe can serve as information storage elements in a dynamic random-access memory device in which decoding and reading the information could be realized by fast electron spin resonance pulses. ${ }^{8}$

In the present paper, it is demonstrated how the molar ratios can affect reactions among precursor solutes and solvents. As an example, copper chloride and melamine are dissolved 
in 1:1 mixture of methanol and dimethyl sulphoxide (DMSO) at room temperature. The molecular structure of melamine is shown in Fig. 1b. It is found that two kinds of large crystals grow at optimized concentrations and molar ratios between copper chloride and melamine. At an optimal concentration of melamine of $0.1 \mathrm{~mol} / \mathrm{L}$, a copper to melamine ratio in the range from 1:1 to 2:1 leads to the formation of a new copper complex composed of all available elements and molecules, i.e. copper, chlorine, melamine, methanol and

DMSO. With a copper to melamine ratio in the range from 3:1 to $4: 1$, both melamine and methanol are passivated, leading to the formation of a coordination polymer with zigzag copper-chlorine chains with each copper coordinated by three chlorines and two DMSO. Both of them are large single crystals with low-dimensional spin structures being candidate materials for magnetic and sensing applications.

\section{Results and discussion}

\section{Synthesis}

All synthesis was carried out at room temperature (around $25{ }^{\circ} \mathrm{C}$ ) by mixing a methanol solution of $\mathrm{CuCl}_{2} \cdot 2 \mathrm{H}_{2} \mathrm{O}$ and a DMSO solution of melamine at different concentrations and molar ratios. The concentrations of $\mathrm{CuCl}_{2} \cdot 2 \mathrm{H}_{2} \mathrm{O}$ tested are $0.8,0.6,0.5,0.4,0.3,0.2,0.1$, 0.05 and $0.025 \mathrm{~mol} / \mathrm{L}$, and the concentrations of melamine are $0.05,0.1$ and $0.2 \mathrm{~mol} / \mathrm{L}$. See Supplementary Information 1 for more details on the synthesis procedures and optimizations. In each case, $2 \mathrm{~mL}$ of a methanol solution of $\mathrm{CuCl}_{2}$ was placed in a $5 \mathrm{~mL}$ glass vial, then $2 \mathrm{~mL}$ of a DMSO solution of melamine was added to the $\mathrm{CuCl}_{2}$ solution. The results are summarized qualitatively in Fig. 1 where 'no crystal' refers to the condition in which no solids are formed while 'not optimized crystal' refers to the condition in which crystals grow but their quantity and/or size are not as large as 'optimized crystal'.

The two optimal conditions are $\left(\rho_{\mathrm{CuCl}_{2}}, \rho_{\text {Melamine }}\right)=(0.2 \mathrm{~mol} / \mathrm{L}, 0.1 \mathrm{~mol} / \mathrm{L})$ for crystals named Cu2M1 and $(0.4 \mathrm{~mol} / \mathrm{L}, 0.1 \mathrm{~mol} / \mathrm{L})$ for crystals named Cu $4 \mathrm{M} 1$, where $\rho_{\mathrm{CuCl}_{2}}$ is the 

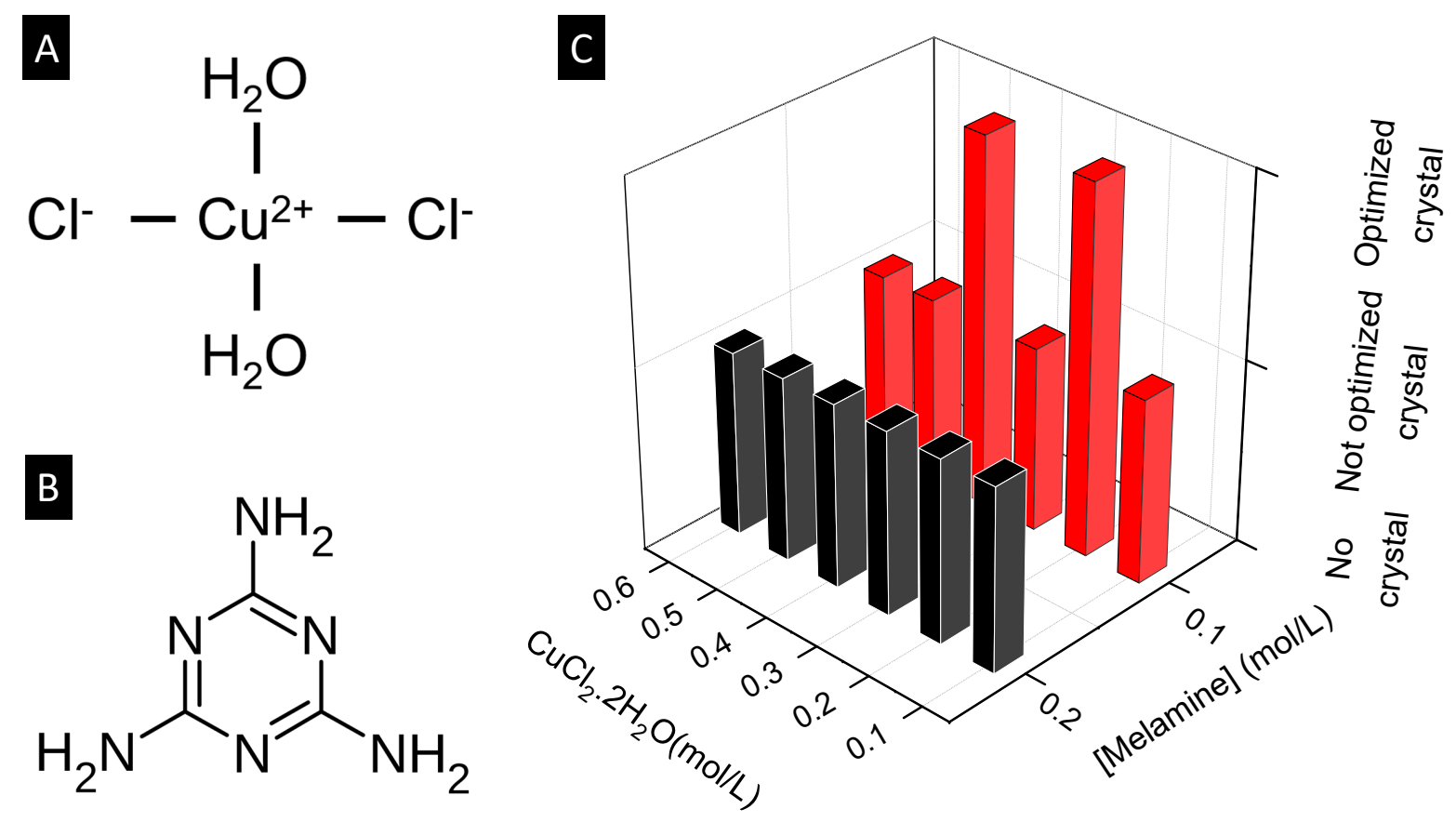

Figure 1: A) The structure of $\mathrm{CuCl}_{2} \cdot 2 \mathrm{H}_{2} \mathrm{O}$. B) The structure of melamine. C) Crystal growth at room temperature with various concentrations of $\mathrm{CuCl}_{2} \cdot 2 \mathrm{H}_{2} \mathrm{O}$ and melamine.

concentration of $\mathrm{CuCl}_{2} \cdot 2 \mathrm{H}_{2} \mathrm{O}$ in methanol and $\rho_{\text {Melamine }}(\mathrm{mol} / \mathrm{L})$ is the concentration of melamine in DMSO. Crystals are formed only within the concentration window $0.05 \mathrm{~mol} / \mathrm{L}$ $\leq \rho_{\mathrm{CuCl}_{2}} \leq 0.6 \mathrm{~mol} / \mathrm{L} \& 0.1 \mathrm{~mol} / \mathrm{L} \leq \rho_{\text {Melamine }} \leq 0.2 \mathrm{~mol} / \mathrm{L}$. The fact that no crystal growth can be achieved at high concentrations of both cations $\mathrm{Cu}(\mathrm{II})$ and anions $\mathrm{Cl}^{-}$can be attributed to high ionic strength that reduces the mobility of the ions in the solution, ${ }^{9}$ hindering the metal to ligand coordination. On the contrary, when the concentration is low, the mobility is high, but the nucleation of the crystal does not occur since the critical nucleation concentration is surpassed, and the collisions between both ligand and metal are less probable. ${ }^{10}$ 


\section{Crystal morphology}

Fig. 2 shows the micrographs of crystals, Cu2M1 and Cu4M1, formed at the optimal conditions. For Cu2M1, dark green rectangular crystals with truncated edges are formed in 5 hours with sizes as large as $350 \mu \mathrm{m}$ in length (see panels A, B and C). As for Cu4M1, light green octagonal crystals as large as $5 \mathrm{~mm}$ across are formed in 48 hours (panels D, E and F). This demonstrates that only doubling the concentration of $\mathrm{CuCl}_{2} \cdot 2 \mathrm{H}_{2} \mathrm{O}$ can drastically alter the morphology of the formed crystal.

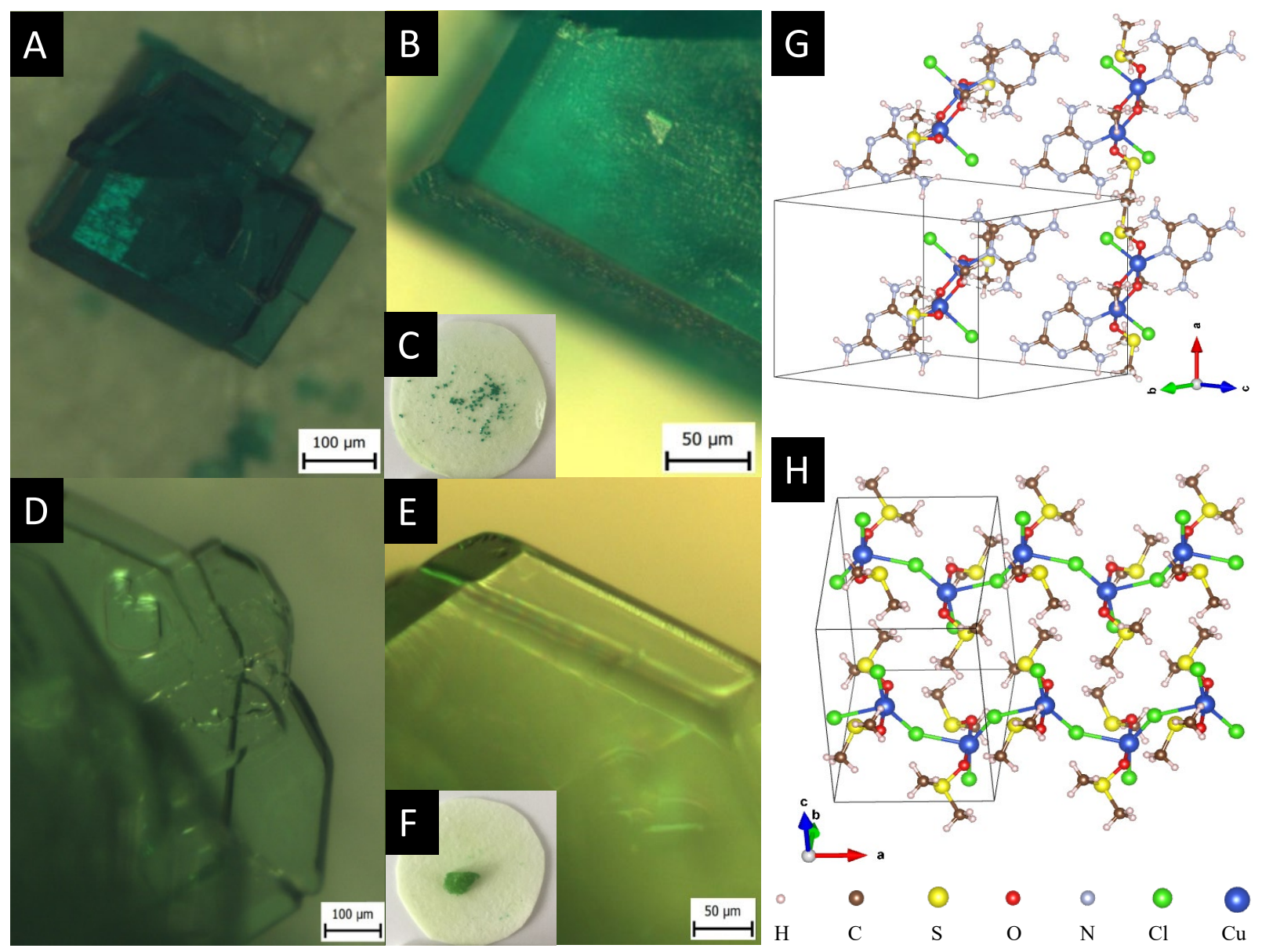

Figure 2: A-C) Optical micrographs of Cu2M1: $\left(\rho_{\mathrm{CuCl}_{2}}, \rho_{\text {Melamine }}\right)=(0.2 \mathrm{~mol} / \mathrm{L}, 0.1 \mathrm{~mol} / \mathrm{L})$. D-F) Optical micrographs of Cu4M1 $\left(\rho_{\mathrm{CuCl}_{2}}, \rho_{\text {Melamine }}\right)=(0.4 \mathrm{~mol} / \mathrm{L}, 0.1 \mathrm{~mol} / \mathrm{L})$. In panels $\mathrm{C}$ and $\mathrm{F}$, crystals are on the membrane with a diameter of about $2.5 \mathrm{~cm}$. G) The structure of Cu2M1 illustrating a layer of hydrogen-bonded melamine molecules in the (lllllane. $\mathrm{H})$ The structure of $\mathrm{Cu} 4 \mathrm{M} 1$ illustrating zigzag $\mathrm{Cu}-\mathrm{Cl}$ chains. 


\section{Structure determination}

\section{Copper coordination polymer Cu4M1}

X-ray diffraction analysis of a Cu4M1 single crystal reveals the structure of a coordination complex (formula $\left.\left[\left(\mathrm{CH}_{3}\right)_{2} \mathrm{SO}\right]_{2} \mathrm{CuCl}_{2}\right)^{1}$, that was previously reported (CCDC deposition number 1142844), ${ }^{11}$ but not much were studied regarding their properties. ${ }^{12,13}$ The determined structure of $\mathrm{Cu} 4 \mathrm{M} 1$, illustrated in Fig. 2, consists of copper, chlorine and DMSO and belongs to the orthorhombic system. As for its structure, it consists of serpent-like $\mathrm{Cu}-\mathrm{Cl}$ chains. Each copper has a trigonal ligand geometry with a crystallographic point group of D3h symmetry, ${ }^{14}$ bonded to two dimethyl sulphoxide molecules through a $\mathrm{Cu}-\mathrm{O}$ bond with a length of $1.95 \mathrm{~A}$, forming a $\mathrm{O}-\mathrm{Cu}-\mathrm{O}$ angle of $173.67^{\circ}$, and to three chlorine atoms in the a-c plane. One of the chlorines is out of the zigzag chain and the other two in the chain. $\mathrm{Cl}-\mathrm{Cu}-\mathrm{Cl}$ angles are $146.46^{\circ}, 112.22^{\circ}$ and $101.32^{\circ}$. The length of the three $\mathrm{Cu}-\mathrm{Cl}$ bonds $(2.75 \mathrm{~A})$ are longer than the covalent $\mathrm{Cu}-\mathrm{Cl}$ bond length $(2.3 \mathrm{~A})$ in $\mathrm{CuCl}_{2}$, indicating the weak covalent bonding. ${ }^{11}$ Figure 3 visualizes the inter- and intramolecular bonds. The bond lengths $[\mathrm{A}]$ and the bond angles $\left[^{\circ}\right]$ are also given. It stands out that the zigzag chain results in a (red marked) "cap". In the area marked in red, two other intramolecular bonds have also been detected (green shaded). The cap encloses a neighbouring strain and it is characterised by several intermolecular interactions (yellow shaded).

\section{Copper-melamine complex Cu2M1}

X-ray diffraction analysis of a single crystal of $\mathrm{Cu} 2 \mathrm{M} 1$ reveals that it is a coordination complex consisting of copper, chloride, melamine, DMSO and methanol, as shown in Fig. 2G. The empirical formula of coordination complex Cu2M1 is $\left[\mathrm{Cu}\left(\mathrm{C}_{3} \mathrm{H}_{6} \mathrm{~N}_{6}\right)\left(\mathrm{OCH}_{3}\right)\left(\left(\mathrm{CH}_{3}\right)_{2} \mathrm{SO}\right) \mathrm{Cl}\right]_{2}$ 2. See Supplementary Information 2 for more details on the structure analysis. To the best

\footnotetext{
${ }^{1}$ CCDC 2061869 contains the supplementary crystallographic data for this paper. These data can be obtained free of charge from The Cambridge Crystallographic Data Centre via www.ccdc.cam.ac.uk/structures

${ }^{2}$ CCDC 2061868 contains the supplementary crystallographic data for this paper. These data can be obtained free of charge from The Cambridge Crystallographic Data Centre via www.ccdc.cam.ac.uk/structures
} 


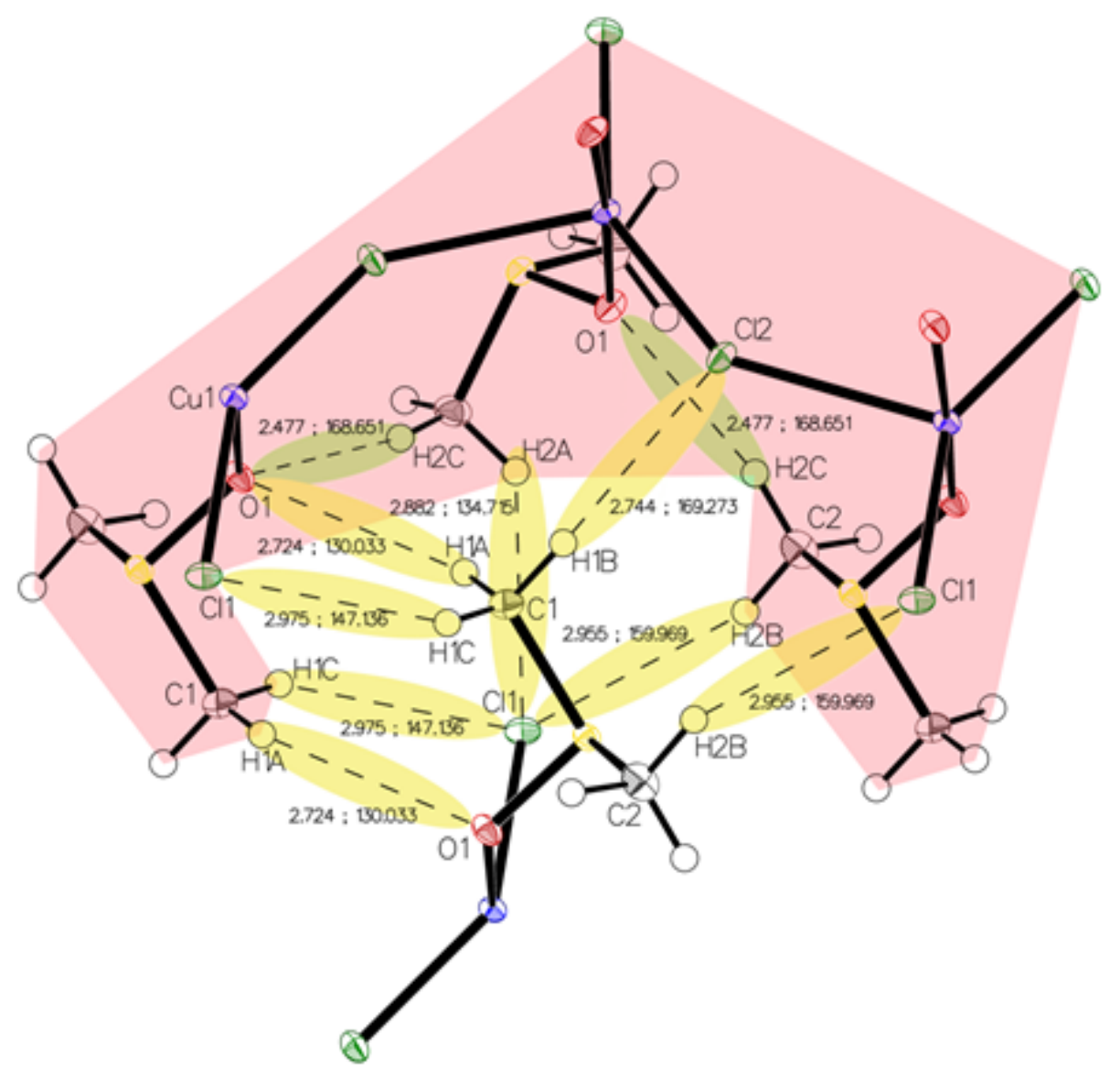

Figure 3: Inter- and intramolecular bonds visualized in the crystal structure of Cu4M1 drawn with $50 \%$ displacement ellipsoid.

of our knowledge, this crystalline structure has not been previously reported, but only those similar to this reported by Chen et al., (2006) (CCDC deposition numbers 280091 and 280092), ${ }^{15}$ Goodgame et al., (1999) with (CCDC deposition number 134810), ${ }^{16}$ and Wiles et al., (2006). ${ }^{17}$ In our Cu2M1 complex, two copper atoms are bridged by the oxygen atoms of two methoxides, forming and angle $\mathrm{O}-\mathrm{Cu}-\mathrm{O}$ of $77.88^{\circ}$. Each copper has a distorted square pyramidal ligand geometry with a crystallographic point group of C4v symmetry. ${ }^{14}$ The top of the square base pyramids is a chlorine $(\mathrm{Cu}-\mathrm{Cl}$ bond length $(2.63 \mathrm{~A})$. The basal plane of the pyramids consists of two $\mathrm{Cu}-\mathrm{O}$ bonds $(1.93 \mathrm{~A})$ by which each copper coordinates with the oxygen of methoxide, another $\mathrm{Cu}-\mathrm{O}$ bond $(1.95 \mathrm{~A})$ which links copper with a molecule of DMSO by its oxygen, and a $\mathrm{Cu}-\mathrm{N}$ bond $(1.98 \mathrm{~A})$ that coordinates copper with a nitrogen 
of the pyridine ring of melamine. The two melamines are in the same plane which is out of the basal plane of the two pyramids. Melamines of adjacent Cu2M1 molecules are hydrogen bonded one another, constituting a global two-dimensional layer of melamine. The packing view along 111 in Fig.4 shows that every dimer (red shaded area) is surrounded by six neighbouring dimers in the plane. The shaded areas within the plane are the seven intermolecular interactions in yellow and the two intramolecular interactions in green.

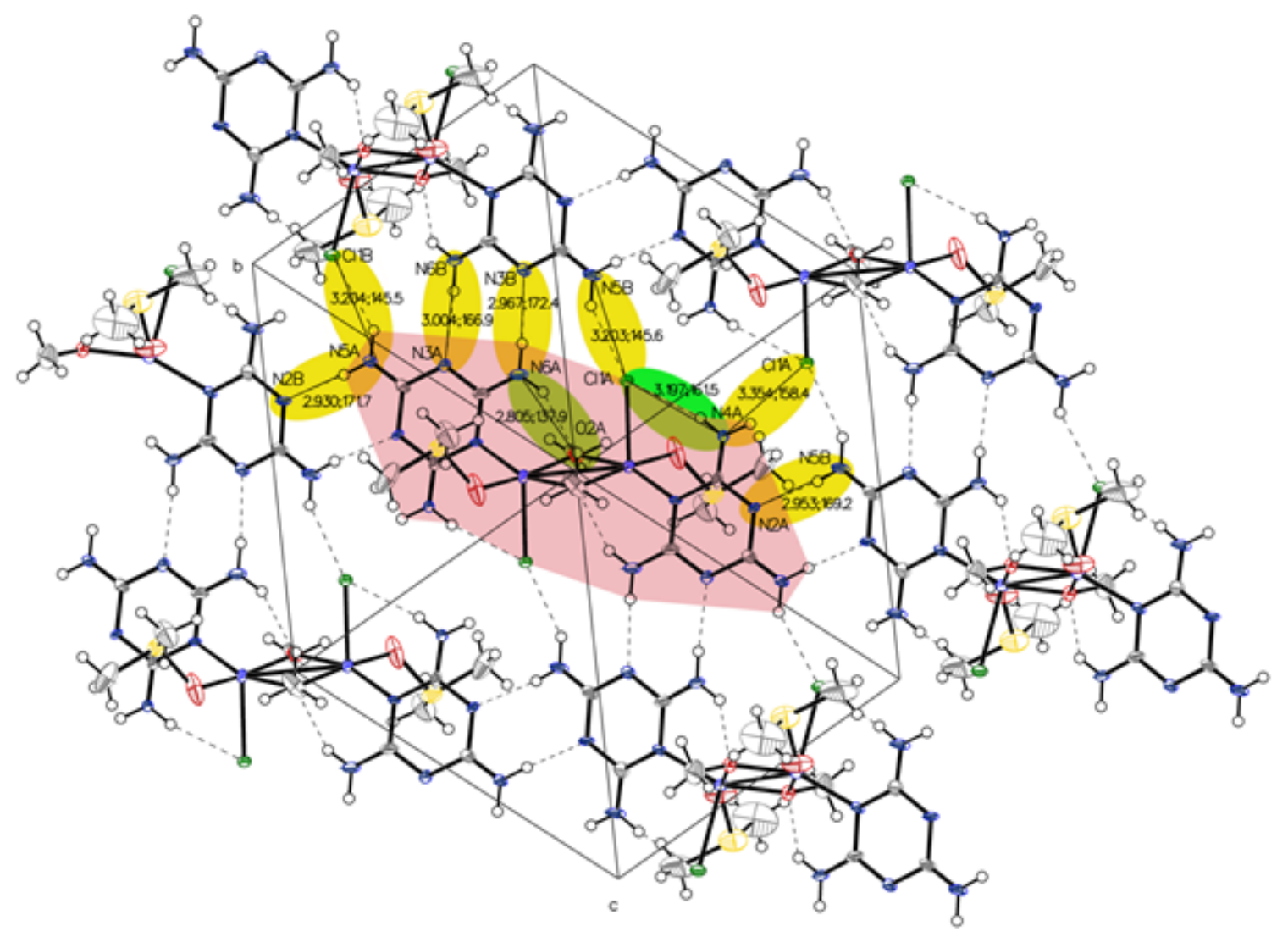

Figure 4: Inter- and intramolecular bonds visualized in the crystal structure of Cu2M1 drawn with $50 \%$ displacement ellipsoid.

Packing of melamine layers along 100 leads to two types of one-dimensional voids accessible by solvents (DMSO and MeOH). Figure 5 compares the structure model for Cu2M1 viewed along the "a" axis without co-crystallised solvents in pannel (a) and the methanol-filled model pictured in pannel (b). The green-shaded void is along the visible radius in pannel 
(a). This barely sterically influenced void allows two different types of solvents (DMSO and $\mathrm{MeOH}$ ) used during the synthesis to co-crystallise in a disordered way. The second type of void (yellow-shaded) is intruded by the coordinated DMSO and limits the available space in which only $\mathrm{MeOH}$ can be modelled. See Supplementary Information 2 for more details.
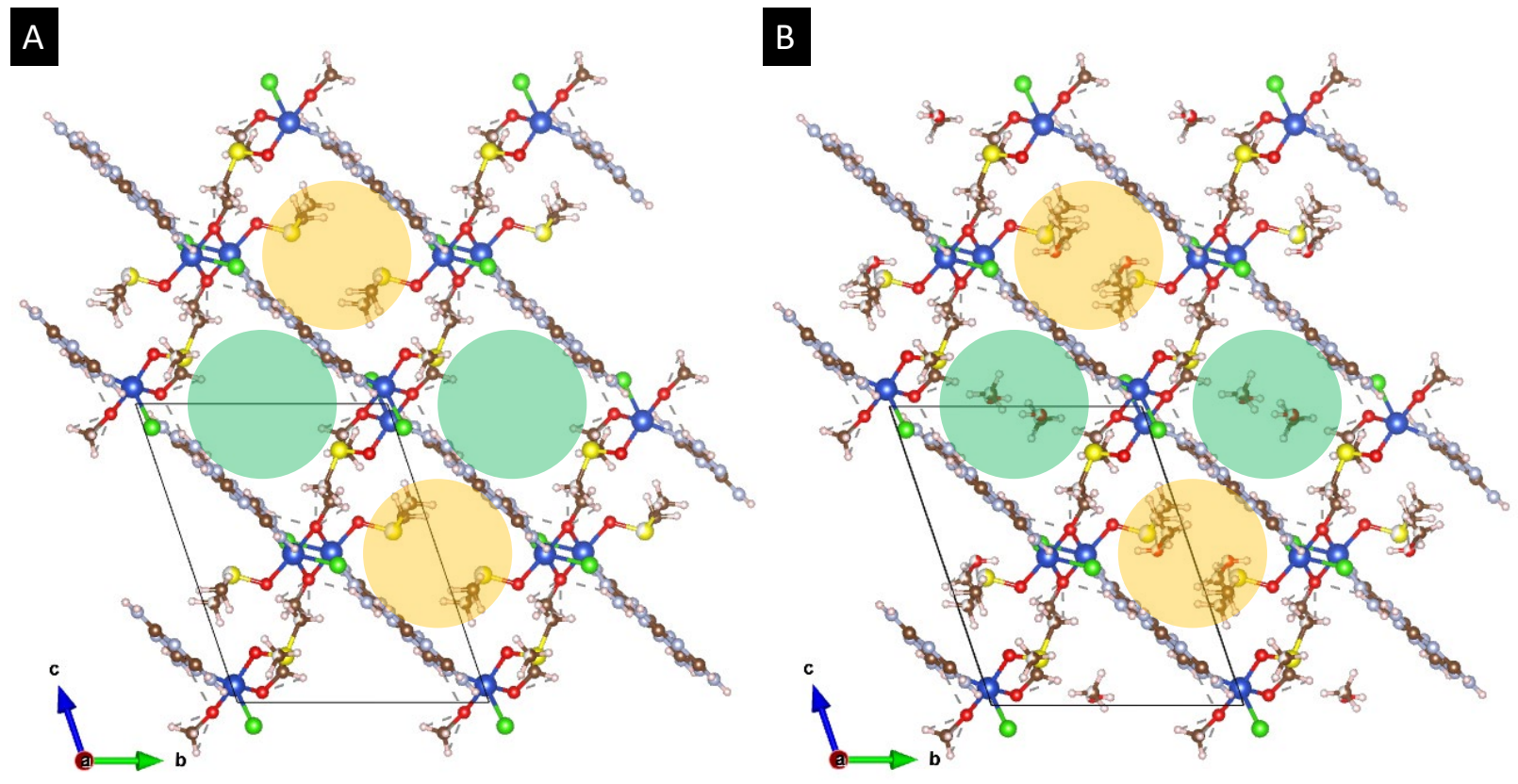

Figure 5: A) The structure of Cu2M1 viewed along the a axis without filling. (B) The structure of Cu2M1 viewed along the a axis with methanol filling. The green and yellowshaded circles represent two types of one-dimensional voids.

\section{Magnetism}

The crystal structures of Cu2M1 and Cu4M1 accommodate low-dimensional coordinations of copper, namely, the dinuclear copper molecular unit in Cu2M1 and the zigzag copper-chlorine chain in $\mathrm{Cu} 4 \mathrm{M} 1$. The low-dimensional nature of the exchange coupling among copper spins are of particular interest towards their potential magnetic applications. 


\section{Copper-melamine complex Cu2M1}

The temperature dependence of the magnetic moment per copper in unit of $\mu_{\mathrm{B}}$ for a dinuclear $\mathrm{Cu}$-melamine complex Cu2M1 sample (33.7 mg of Cu2M1 crystals encapsulated in a gelatin capsule) in an applied magnetic field of $1 \mathrm{~T}$ is shown in Fig. 6A. As planar crystals are stacked horizontally, the magnetic field is applied normal to the crystal plane for the majority of crystals. The net magnetism of dinuclear Cu-melamine complex is much smaller than the dashed curve labelled as Curie's Law for a paramagnetic $1 / 2$ spin with an effective

magnetic moment of $\mu_{\mathrm{B}}=g_{S} \sqrt{S(S+1)} \mu_{\mathrm{B}} \simeq 1.73 \mu_{\mathrm{B}}$, indicating the presence of a strong antiferromagnetic coupling. The magnetic susceptibility as a function of temperature can be analysed using the Bleaney Bowers equation for a exchange-coupled pair of $\mathrm{S}=1 / 2$ spins ${ }^{18} 19$

$$
\chi_{\mathrm{Cu}}=\frac{g_{S}^{2} \mu_{B}^{2}}{k_{B}\left(T-T_{w}\right)} \frac{1}{3+e^{-2 J / k_{B}\left(T-T_{w}\right)}}(1-\zeta)+\frac{g_{S}^{2} \mu_{B}^{2}}{4 k_{B}\left(T-T_{w}\right)} \zeta+\eta
$$

where, the first, second and third terms are the Bleaney Bowers equation, Curie-Weiss law taking into account paramagnetic impurities and temperature-independent constant $\eta$, respectively. Here, $g_{S} \simeq 2$ is the electron spin g-factor, $k_{B}$ the Boltzmann constant, $J$ the exchange energy between the spins and $\zeta$ corresponds to a concentration of $1 / 2$ paramagnetic impurities, e.g. isolated $\mathrm{Cu}(\mathrm{II})$ on defects and surfaces. Weiss temperature $T_{w}$ takes the moleular field due to intermolecular exchange into account. A least-square fitting is satisfactory with $J=-0.0542 \pm 0.0047 \mathrm{eV}\left(\simeq-437 \mathrm{~cm}^{-1}\right), T_{w}=-1.98 \pm 0.05[\mathrm{~K}], \zeta=0.158 \pm 0.002$ and $\eta=8.237 \times 10^{-4} \pm 2.11 \times 10^{-5}\left[\mu_{B} / \mathrm{Cu}\right]$. In Fig.6A, the Bleaney Bowers fit magnified 1000 times is plotted. Because of the relatively high exchange constant, the Bleaney Bowers component is small compared with the Curie-Weiss term in the measured temperature range $2-300 \mathrm{~K}$ and reachs only about a half of the Curie's component at room temperature. A negative Weiss temperature of $T_{w}=-1.98 \pm 0.05[\mathrm{~K}]$ can be attributed to intermolecular anti-ferromagentic coupling. 


\section{Copper coordination polymer Cu4M1}

As shown in Fig. 6B, the magnetization of a planar single crystal of copper coordination polymer Cu4M1 (24.0 mg) in the magnetic field applied normal to the crystal plane exhibits a maximum of $0.0285 \mu_{\mathrm{B}}$ at $10 \mathrm{~K}$. Provided that the exchange coupling is anisotropic and strong along the zigzag copper-chlorine-copper chain, the temperature dependence of the magnetic susceptibility can be evaluated based on exchange Hamiltonian $\hat{\mathrm{H}}=-\sum_{i j} \mathrm{~J}_{i j} \hat{\mathrm{S}}_{i} \hat{\mathrm{S}}_{j}$ taking exchange coupling between any two adjacent spins in a one-dimensional spin chain

into account. ${ }^{20212223} .{ }^{24}$ The Bonner-Fisher equation derived from the above Hamiltonian for a $S=1 / 2$ Heisenberg chain ${ }^{2526}$ together with a paramagnetic term, eq.2, is as follows.

$$
\chi_{C u}=\frac{g_{S}^{2} \mu_{B}^{2}}{12 k_{B} T} \frac{1+u(K)}{1-u(K)}(1-\zeta)+\frac{g_{S}^{2} \mu_{B}^{2}}{4 k_{B} T} \zeta,
$$

where $u(K)=\operatorname{coth}(K)-(1 / K)$ and $K=J / 2 k_{B} T$, and $\zeta$ corresponds to a concentration of $1 / 2$ paramagnetic impurities or the inverse-temperature term that arises from staggered spins. ${ }^{27}$ The least-square fit reproduces the experimental temperature dependence very well as shown in Fig.6b, giving rise to $g=2.331 \pm 0.005, J=-3.05 \pm 0.02 \mathrm{meV}\left(24.6 \mathrm{~cm}^{-1}\right)$, $\zeta=0.056 \pm 0.0011 . \mathrm{g}$ is quite larger than 2 for pure spin states, but this value depends largely on the normalization of the data that may contain errors.

\section{Synthesis of copper coordination polymer Cu4M1 without melamine}

The structure analysis reveals that with a $\mathrm{Cu}$-melamine ratio of $4: 1\left(0.4 \mathrm{~mol} / \mathrm{L}\right.$ of $\mathrm{CuCl}_{2} \cdot 2 \mathrm{H}_{2} \mathrm{O}$ in methanol and $0.1 \mathrm{~mol} / \mathrm{L}$ of melamine in DMSO) copper coordination polymer Cu4M1 is formed despite the presence of melamine. During their synthesis at room temperature, the pH of the media evolves differently in the 2:1 and 4:1 mixed solutions. ${ }^{28}$ Although evaluating $\mathrm{pH}$ values of organic and aprotic solvents is a complicated task, their relative changes upon chemical reactions are worth to be noted. Since there is no $\mathrm{OH}^{-}$in the precursor solvents, the $\mathrm{pH}$ is only related with the presence of $\mathrm{H}^{+}$. The $\mathrm{pH}$ value of $0.2 \mathrm{~mol} / \mathrm{L}$ of $\mathrm{CuCl}_{2} \cdot 2 \mathrm{H}_{2} \mathrm{O}$ in 

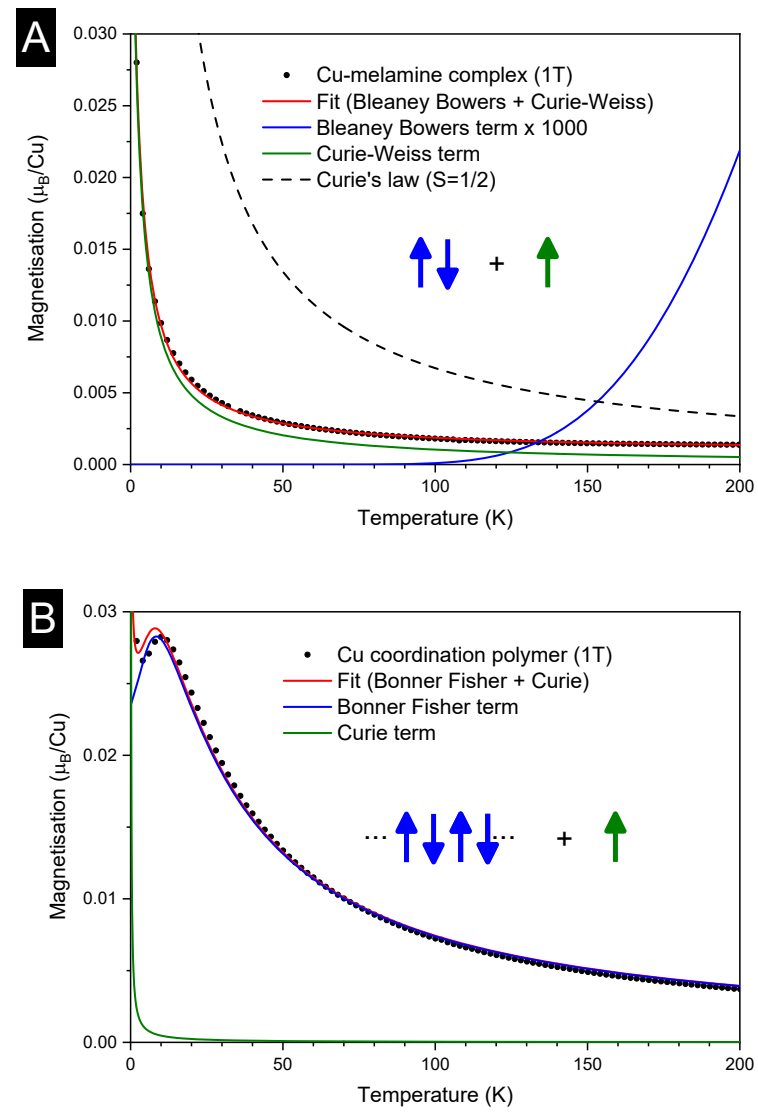

Figure 6: Magnetisation versus temperature for (A) dinuclear Cu-melamine complex Cu2M1 and (B) 1D-Cu coordination polymer Cu4M1

methanol, $0.4 \mathrm{~mol} / \mathrm{L}$ of $\mathrm{CuCl}_{2} \cdot 2 \mathrm{H}_{2} \mathrm{O}$ in methanol and $0.1 \mathrm{~mol} / \mathrm{L}$ of melamine before mixing are $1.03 \pm 0.03,0.62 \pm 0.04$ and $9.64 \pm 0.02$, respectively. The $\mathrm{pH}$ of the $2: 1$ and $4: 1$ solutions just after mixing are $5.96 \pm 0.03$ and $5.56 \pm 0.02$, respectively. After the formation of crystals of dinuclear copper-melamine complex $\mathrm{Cu} 2 \mathrm{M} 1$, the $\mathrm{pH}$ of the 2:1 solution remains unchanged within the confidence interval $(5.94 \pm 0.03)$. (This statistical parameter is determined by a "t of Student" distribution with $95 \%$ confidence interval, for which it is considered five simultaneous measurements.) On the contrary, the $\mathrm{pH}$ of the 4:1 solution is increased to $6.14 \pm 0.03$ after the formation of crystals of copper coordination polymer Cu4M1. This increase can be attributed to a reduction of $\mathrm{H}^{+}$as a result of protonation of melamine which is initially deprotonated in pure DMSO. This leaves passivated neutral melamine which doesn't get coordinated with $\mathrm{Cu}(\mathrm{II})$ ions. Likewise, the $\mathrm{pH}$ barely changes in the 
2:1 solution because melamine ions react with $\mathrm{Cu}(\mathrm{II})$ before being protonated. Hence, the proton concentration needs to be optimised for the formation of copper coordination polymer Cu4M1.

In order to justify the above-mentioned scenario, the synthesis of copper coordination polymer Cu4M1 has been attempted by mixing a methanol solution of $\mathrm{CuCl}_{2} \cdot 2 \mathrm{H}_{2} \mathrm{O}$ with aprotic DMSO whose $\mathrm{pH}$ value adjusted by adding anhydrous acetic acid. It is found that crystals of the same copper coordination polymer are formed in the solution without melamine when the $\mathrm{pH}$ is adjusted to $9.64 \pm 0.02$, while no crystals are formed without acetic acid. Thus, the concentration of proton plays an important role in the coordination of DMSO and chlorine with copper ions in the presence of methanol.

\section{Conclusions}

The novel copper-melamine complex and the copper coordination polymer have been synthesized selectively by adjusting the concentrations of copper (II) chloride dihydrate and melamine in a mixed solution of methanol and DMSO at room temperature. Crystals of copper-melamine complex Cu2M1 formed with a Cu-melamine ratio of 2:1 has a green square shape with sizes as large as $350 \mu \mathrm{m}$ across. The hydrogen-bonded two-dimensional lattice is composed of planes of melamine and one-dimensional pores along the a-axis that accommodate solvent molecules. Crystals of copper coordination polymer Cu4M1 formed with a $\mathrm{Cu}$-melamine ratio of 4:1 are light green gems-like octagonal and can grow as large as $5 \mathrm{~mm}$ across. The lattice is composed of $\mathrm{Cu}-\mathrm{Cl}$ zigzag chains and has no porosity. Both $\mathrm{Cu} 2 \mathrm{M} 1$ and Cu4M1 exhibit low-dimensional magnetic properties. The magnetic susceptibility of Cu2M1 can be modelled well based on the Hamiltonian for paired spins of $1 / 2$ and that of Cu4M1 based on a spin-1/2 anti-ferromagnetic Ising chain. The well-controlled synthesis of the quality and large monocrystals demonstrated in the present study will pave the way for future research on spintronic applications of inorganic and organic-inorganic hybrid 
materials.

\section{Experimental}

\section{Acknowledgement}

H.S. acknowledges support from the Austrian Science Fund (FWF) P30431-N36 and the Czech Science Foundation (GACR) project 19-15217S. This work was supported in part by the Austrian Federal Ministry of Education, Science and Research (BMBWF), OeAD-GmbH and the Ministry of Education, Youth and Sports (MEYS) of the Czech Republic, through Scientific \& Technological Cooperation (WTZ) program, No. CZ 18/2019 and 8J19AT026.

\section{Supporting Information Available}

The following files are available free of charge.

- cu-melamine-ESI1: Experimental and synthesis details

- cu-melamine-ESI2: Structure analysis

\section{References}

(1) Cleaves, H. J. Encyclopedia of Astrobiology; Springer Berlin Heidelberg: Berlin, Heidelberg, 2011; pp 1183-1183.

(2) Campbell, M. G.; Sheberla, D.; Liu, S. F.; Swager, T. M.; Dincă, M. Cu3(hexaiminotriphenylene)2: An Electrically Conductive 2D Metal-Organic Framework for Chemiresistive Sensing. Angewandte Chemie International Edition 2015, 54, $4349-4352$. 
(3) Kitagawa, S.; Kitaura, R.; Noro, S. I. Functional porous coordination polymers. Angewandte Chemie - International Edition 2004, 43, 2334-2375.

(4) Kostakis, G. E. Structural Design and Properties of Coordination Polymers; 2018.

(5) Williams, K. A.; Boydston, A. J.; Bielawski, C. W. Main-chain organometallic polymers: synthetic strategies, applications, and perspectives. Chemical Society Reviews 2007, 36, 729.

(6) Glover, T. G.; Walton, K. S. Water Stability of Metal-Organic Frameworks. Gas Adsorption in Metal-Organic Frameworks 2018, 363-392.

(7) Yuan, S. et al. Stable Metal-Organic Frameworks: Design, Synthesis, and Applications. Advanced Materials 2018, 30, 1-35.

(8) Leuenberger, M. N.; Loss, D. Quantum computing in molecular magnets. Nature 2001, 410, 789-793.

(9) Peng, W.; Li, H.; Liu, Y.; Song, S. A review on heavy metal ions adsorption from water by graphene oxide and its composites. Journal of Molecular Liquids 2017, 230, 496-504.

(10) Stock, N.; Biswas, S. Synthesis of metal-organic frameworks (MOFs): Routes to various MOF topologies, morphologies, and composites. Chemical Reviews 2012, 112, 933-969.

(11) Willett, R. D.; Chang, K. The crystal structure of copper(II) chloride bis(dimethylsulphoxide). Inorganica Chimica Acta 1970, 4, 447-451.

(12) Becker, S.; Dürr, M.; Miska, A.; Becker, J.; Gawlig, C.; Behrens, U.; IvanovićBurmazović, I.; Schindler, S. Copper Chloride Catalysis: Do $\mu 4$-Oxido Copper Clusters Play a Significant Role? Inorganic Chemistry 2016, 55, 3759-3766. 
(13) Chebbi, H.; Chebbi, M.; Guesmi, A.; Arfaoui, Y. Crystal structure determination, and DFT Calculations of dichlorobis-(dimethylsulfoxide-O)copper(II). Journal of Structural Chemistry 2016, 57, 1104-1110.

(14) Flurry, R. Symmetry Groups; Prentice-Hall, 1980; pp 115-127.

(15) Chen, C.; Yeh, C. W.; Chen, J. D. Syntheses, structures and thermal properties of two new copper(II) melamine complexes. Polyhedron 2006, 25, 1307-1312.

(16) Goodgame, D. M. L.; Hussain, I.; White, A. J. P.; Williams, D. J. Cu - melamine coordination. 1999, 2899-2900.

(17) Wiles, A. B.; Bozzuto, D.; Cahill, C. L.; Pike, R. D. Copper (I) and (II) complexes of melamine. Polyhedron 2006, 25, 776-782.

(18) Bleaney, B. Anomalous paramagnetism of copper acetate. Reviews of Modern Physics 1953, 25, 161-162.

(19) Figgis, B.; Martin, R. Magnetic studies with copper (II) salts.1. anomalous paramagnetism and delta-bonding in anhydrous and hydrated copper (II) acetates. Journal of the Chemical Society 1956, 3837-3846.

(20) Bonner, J.; Fisher, M. Linear magnetic chains with anisotropic coupling. Physical Review 1964, 135, A640.

(21) Hall, J.; Marsh, W.; Weller, R.; Hatfield, W. Exchange coupling in the alternating -chain compounds catena -di-mu-chloro-bis(4-methylpyridine) copper (II), catena-dimu-bromo-bis(N-methylimidazole) copper(II), catena -[hexanedione bis (thiosemicarbazonato)]copper(II), and catena -[octanedione "bis(thiosemicarbazonato)]copper(II). Inorganic Chemistry 1981, 20, 1033-1037.

(22) Borrasalmenar, J.; Coronado, E.; Curely, J.; Georges, R.; Gianduzzo, J. Alternating 
chains with ferromagnetic and antiferromagnetic interactions- Theory and magnetic -properties. Inorganic Chemistry 1994, 33, 5171-5175.

(23) Dalai, S.; Mukherjee, P.; Rogez, G.; Mallah, T.; Drew, M.; Chaudhuri, N. Synthesis, crystal structures, and magnetic properties of two new 1D copper(II) coordination polymers containing fumarate(-2) and chelating N,N '-donor as ligands. European Journal of Inorganic Chemistry 2002, 3292-3297.

(24) Marino, N.; Armentano, D.; De Munno, G.; Cano, J.; Lloret, F.; Julve, M. Synthesis, Structure, and Magnetic Properties of Regular Alternating mu-bpm/di-mu-X Copper(II) Chains (bpm=2,2 '-bipyrimidine; X = OH, F). Inorganic Chemistry 2012, 51, $4323-4334$.

(25) Fisher, M. E. Magnetism in One-Dimensional Systems-The Heisenberg Model for Infinite Spin. American Journal of Physics 1964, 32, 343-346.

(26) Estes, W. E.; Gavel, D. P.; Hatfield, W. E.; Hodgson, D. J. Magnetic and structural characterization of dibromo- and dichlorobis(thiazole) copper(II). Inorg. Chem. 1978, $17,1415-1421$.

(27) Feyerherm, R.; Abens, S.; Günther, D.; Ishida, T.; Meißner, M.; Meschke, M.; Nogami, T.; Steiner, M. Magnetic-field induced gap and staggered susceptibility in the $\mathrm{S}=1 / 2$ chain $[\mathrm{PM} \cdot \mathrm{Cu}(\mathrm{NO} 3) 2 \cdot(\mathrm{H} 2 \mathrm{O}) 2] \mathrm{n}(\mathrm{PM}=$ pyrimidine $)$. Journal of Physics: Condensed Matter 2000, 12, 8495-8509.

(28) Ostwald, W. Zeitschrift für Physikalische Chemie; 1900; Vol. 34U; pp 495-503. 


\section{Graphical TOC Entry}

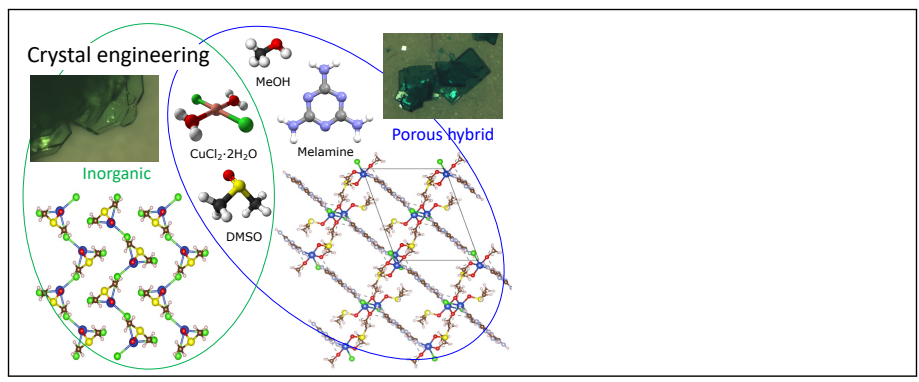

